\title{
Numerical Study of the Dynamics of Laser Lineshape and Linewidth
}

\author{
M. ESKEF* \\ Department of Physics, Atomic Energy Commission of Syria, P.O. Box 6091, Damascus, Syria \\ (Received January 28, 2015; revised version May 31, 2016; in final form July 27, 2016)
}

\begin{abstract}
A rate equations model for lasers with homogeneously broadened gain is written and solved in both time and frequency domains. The model is applied to study the dynamics of laser lineshape and linewidth using the example of He-Ne laser oscillating at $\lambda=632.8 \mathrm{~nm}$. Saturation of the frequency spectrum is found to take much longer time compared to the saturation time of the overall power. The saturated lineshape proves to be Lorentzian, whereas the unsaturated line profile is found to have a Gaussian peak and a Lorentzian tail. Above threshold, our numerical results for the linewidth are in good agreement with the Schawlow-Townes formula. Below threshold, however, the linewidth is found to have an upper limit defined by the spectral width of the pure cavity. Our model provides a unique and powerful tool for studying the dynamics of the frequency spectrum for different kinds of laser systems, and is also applicable for investigating lineshape and linewidth of pulsed lasers.
\end{abstract}

DOI: 10.12693/APhysPolA.130.710

PACS/topics: 42.55.Ah, 42.55.Lt

\section{Introduction}

Laser lineshape and linewidth play a key role in theoretical studies related to the fields of resonance ionization spectroscopy (RIS) [1, 2], laser isotope separation [3], as well as resonance ionization mass spectrometry (RIMS) [4]. The first treatment of laser linewidth was conducted by Schawlow and Townes in the late 1950s. They considered the laser as a selective amplifier with a noise source due to the spontaneously emitted photons, and found that the linewidth decreases in inverse proportion to the laser power [5]. Extremely small widths of the order of a few $\mathrm{Hz}$ were predicted for reliable values of the laser power. Later on, several approaches have been used to develop a comprehensive laser theory capable of describing lineshape and linewidth of the laser radiation. In Lamb's laser theory, the laser field is described by the classical Maxwell equations and the active atoms are accounted for by a nonlinear macroscopic polarization vector in phase with the field $[6,7]$. In the quantum noise theory developed by Lax et al. the laser is treated as a rotating-wave Van der Pol oscillator with a quantum noise source due to spontaneous emission $[8,9]$. Haken's quantum statistical theory of laser starts with the Schrödinger equation for the laser system plus its environment, reduces that equation in a first step to the master equation for the density operator and transforms the latter operator equation, further on, into a FokkerPlanck equation for the probability density of the complex laser field amplitude [10].

Actually, all these models have two characteristics in common: first, they provide a solution for the lineshape and linewidth under steady state conditions, i.e. they

*e-mail: pscientific@aec.org.sy give no information about the dynamics of lineshape and linewidth. Second, their way of calculating lineshape and linewidth leads through the implicit assumption that the laser field can be represented by a complex amplitude oscillating at the resonance frequency of the pure cavity, which is equivalent to requiring the solutions for the laser field to be self-consistent. In this context, the finite linewidth of the laser radiation is not related to the existence of solutions at frequencies other than the resonance frequency, it is just the fingerprint of the statistical fluctuations of the complex amplitude when transformed into the frequency domain. The Fokker-Plank equation divides these statistical fluctuations into a drift term and a diffusion term. The former is assigned the coherent amplitude fluctuations related to the cavity loss and the amplification by stimulated emission, whereas the latter term is assigned the incoherent phase fluctuations due to spontaneous emission [11]. Under steady state conditions the drift term is set to zero, whereas the diffusion term, which describes a temporal phase noise caused by spontaneously emitted photons, is translated into a spectral line profile by means of the Wiener-Kintchine theorem.

Recently, an alternative, quite different approach to calculating laser lineshape and linewidth was presented by the author of this study [12]. This alternative approach is based on extending Einstein's laser rate equations into the frequency domain and solving the time- and frequency-dependent rate equations free from the artificial boundaries connected with requiring self-consistency of the laser field. Promising results have been achieved for homogeneously, as well as for inhomogeneously broadened gain profile. The lineshape was proved to follow a Lorentzian; the linewidth was found to saturate at a value close to the cavity width in the case of inhomogeneous gain, and to continue decreasing to very small values limited only by the finite resolution of the numerical calculation in the case of homogeneous gain. 
However, in order to make the rate equations model of [12] capable of producing quantitative rather than qualitative results on the dynamics of laser lineshape and linewidth for a laser with homogeneously broadened gain, further improvement needs to be attained on both the physical as well as the technical level. Addressing this task is the main subject of the present work. A detailed description of the improved model is given in the next section highlighting the changes made to the rate equations of [12]. Afterwards, the model is applied to investigate the dynamics of laser lineshape and linewidth, as well as the relationship between the linewidth and the laser power. Results are discussed in comparison with theoretical as well as experimental data available in the literature. We will refer to the example of $\mathrm{He}-\mathrm{Ne}$ laser oscillating at $\lambda=632.8 \mathrm{~nm}$, however, the concept of the model is universal and can, therefore, be considered applicable to other kinds of lasers with homogeneously broadened gain.

\section{The model}

Our rate equations follow the main lines of the common concept used by Burak et al. for small gain lasers [13], as well as by Rigrod [14] and Kaufman et al. [15] for high gain lasers. However, our treatment distinguishes itself from earlier works in two aspects: (a) we write and solve the laser rate equations in both time and frequency domains; and (b) we incorporate the spectral effect of the cavity into the laser rate equations instead of adopting predefined cavity modes. As explained in [12], these two additions give our model the capability of providing an independent calculation of the dynamics of the laser frequency spectrum in contrast to conventional rate equations models which are restricted to the dynamics of the integral intensities of a few predefined modes.

Within the framework of an idealized four-level formalism, which is quite sufficient for the purpose of the present study, our set of rate equations consists of two equations for the rate of change of the population density $M_{1,2}$ of the lower, respectively the upper laser state, as well as a third equation describing the rate of change of the spectral density $n(\nu) \mathrm{d} \nu$ of photons propagating along the optical axis inside the cavity. The first two rate equations read as follows:

$$
\begin{aligned}
& \frac{\mathrm{d}}{\mathrm{d} t} M_{1}=\beta+A_{21} M_{2}-A_{10} M_{1}, \\
& \frac{\mathrm{d}}{\mathrm{d} t} M_{2}=-\beta-\left(A_{21}+A_{20}\right) M_{2}+\Phi,
\end{aligned}
$$

where $\beta$ is the rate of stimulated emission, $A_{21}$ is the Einstein coefficient for spontaneous decay of the upper state into the lower one, $A_{10}$ and $A_{20}$ are the decay rates of the lower, respectively the upper state into the ground state, and $\Phi$ is the pumping rate. The third equation expresses the fact that the rate of change of the photon spectral density inside the cavity is the sum of three effects: the spontaneous emission, the stimulated emission, and the cavity losses

$$
\frac{\mathrm{d}}{\mathrm{d} t} n(\nu) \mathrm{d} \nu=\dot{n}_{\mathrm{sp}}(\nu) \mathrm{d} \nu+\dot{n}_{\mathrm{st}}(\nu) \mathrm{d} \nu+\dot{n}_{\mathrm{c}}(\nu) \mathrm{d} \nu .
$$

Spontaneously emitted photons follow, in general, a Lorentzian distribution $\mathcal{L}(\nu) \mathrm{d} \nu$ characterized by the transition frequency $\nu_{0}$ and the homogeneous width $\Gamma_{\mathrm{h}}$. However, the quantum electrodynamics tells us that the probability for emitting a photon is proportional to the intensity of the electromagnetic wave associated with that photon [16]. For a photon being emitted along the optical axis of the cavity, the associated wave will undergo multiple reflections on the cavity mirrors, and the intensity will experience an enhancement/inhibition by a cavity specific, frequency sensitive factor $\mathcal{A}_{\mathrm{c}}(\nu)$ due to constructive/destructive single photon interference [12]. Therefore, the first term on the right hand side of Eq. (3) can be written as

$$
\dot{n}_{\mathrm{sp}}(\nu) \mathrm{d} \nu=A_{21} M_{2} \xi \frac{l_{\mathrm{m}}}{l_{\mathrm{c}}} \mathcal{L}(\nu) \mathrm{d} \nu \mathcal{A}_{\mathrm{c}}(\nu),
$$

where $\xi$ is a geometrical factor accounting for the limited solid angle of the optical axis, $l_{\mathrm{m}} / l_{\mathrm{c}}$ is the ratio of the active medium length to the cavity length. Considering the cavity as a Fabry-Perot resonator, characterized by its length $l_{\mathrm{c}}$ and the mirror reflectivities $R_{1}$ and $R_{2}$, the cavity specific enhancement factor $\mathcal{A}_{\mathrm{c}}(\nu)$, to be referred to in the following as the spectral function of the cavity, can be calculated as

$$
\mathcal{A}_{\mathrm{c}}(\nu)=\frac{1}{1+R_{1} R_{2}-2 \sqrt{R_{1} R_{2}} \cos \delta(\nu)},
$$

where $\delta(\nu)$ refers to the round trip phase shift given by $\delta(\nu)=4 \pi l_{\mathrm{c}} \nu / c[12]$. Note that the enhancement of spontaneous emission when the emitting atoms/molecules are located inside a cavity was first reported by Purcell as early as in the 1940s, and is since then known as the Purcell effect. According to the original work of Purcell, the enhancement factor, also referred to as the Purcell factor, is proportional to the quality factor of the cavity [17]. With this in mind, our spectral function of the cavity $\mathcal{A}_{\mathrm{c}}(\nu)$, given by Eq. (5), may be considered as our ansatz for the frequency dependent Purcell factor.

The second term on the right hand side of Eq. (3) describes the contribution of stimulated emission to the rate of change of $n(\nu) \mathrm{d} \nu$, and can be related to the single pass gain $G(\nu)$ by considering the propagation of the beam along the optical axis inside the cavity. The beam passes through the active medium tube, on the average, once each half round trip time $l_{\mathrm{c}} / c$ being thereby amplified by $G(\nu)$, and hence, the rate of change of $n(\nu) \mathrm{d} \nu$ due to stimulated emission can be written as follows:

$$
\dot{n}_{\mathrm{st}}(\nu) \mathrm{d} \nu=\frac{c}{l_{\mathrm{c}}}[G(\nu)-1] n(\nu) \mathrm{d} \nu .
$$

The single pass gain $G(\nu)$ is a function of the population inversion and can be approximated by

$$
G(\nu)=\exp \left(\left(g_{1} M_{2}-g_{2} M_{1}\right) \sigma_{\mathrm{c}}(\nu) l_{\mathrm{m}}\right),
$$

where $g_{1}$ and $g_{2}$ are the degeneracy factors of the lower and upper states, respectively, and $\sigma_{\mathrm{c}}(\nu)$ is the crosssection for a photon with the frequency $\nu$, propagating along the optical axis of the cavity, to stimulate a transition from the upper into the lower state. 
The cavity dependent stimulated emission crosssection $\sigma_{\mathrm{c}}(\nu)$ has the form

$$
\sigma_{\mathrm{c}}(\nu)=\sigma_{0} \frac{\Gamma_{\mathrm{h}}^{2}}{4\left(\nu-\nu_{0}\right)^{2}+\Gamma_{\mathrm{h}}^{2}} \mathcal{A}_{\mathrm{c}}(\nu),
$$

where $\sigma_{0}$ is the stimulated transition cross-section at $\nu_{0}$, the Lorentzian factor accounts for the deviation from the central frequency $\nu_{0}$, and the spectral function of the cavity $\mathcal{A}_{\mathrm{c}}(\nu)$ accounts for the enhancement/inhibition of the emission probability due to single photon interference.

The third term on the right hand side of Eq. (3) represents the rate of change of $n(\nu) \mathrm{d} \nu$ due to cavity losses, and is well approximated by the mean cavity loss over a round trip time $2 l_{\mathrm{c}} / c$. During a round trip, the beam is reflected once on each of the two mirrors, the corresponding loss of $n(\nu) \mathrm{d} \nu$ is given by $\left(1-R_{1} R_{2}\right) n(\nu) \mathrm{d} \nu$, and accordingly, the rate of change of $n(\nu) \mathrm{d} \nu$ due to the cavity losses can be set to

$$
\dot{n}_{\mathrm{c}}(\nu) \mathrm{d} \nu=-\frac{c}{2 l_{\mathrm{c}}}\left(1-R_{1} R_{2}\right) n(\nu) \mathrm{d} \nu .
$$

Finally, the overall rate of stimulated emission $\beta$ can be derived by integrating $\dot{n}_{\text {st }}(\nu) \mathrm{d} \nu$ over all frequencies

$$
\beta=\frac{l_{\mathrm{c}}}{l_{\mathrm{m}}} \int_{\nu} \dot{n}_{\mathrm{st}}(\nu) \mathrm{d} \nu,
$$

where the factor in front of the integral accounts for the fact that photons are distributed along the full cavity length $l_{\mathrm{c}}$, whereas stimulated emission takes place only along the length of the active medium tube $l_{\mathrm{m}}$. Making use of Eq. (6), the rate of stimulated emission can be written as follows:

$$
\beta=\frac{c}{l_{\mathrm{m}}} \int_{\nu}[G(\nu)-1] n(\nu) \mathrm{d} \nu .
$$

With the aid of Eqs. (4)-(10), the set of rate Eqs. (1)-(3) can be solved by means of numerical integration. The results provide an insight into the dynamics of the laser frequency spectrum which is the main subject of the present study. In addition, special focus will also be placed on the relationship between linewidth and power. The latter is related to the frequency-integrated photon density according to

$$
P=h \nu_{0} c\left(1-R_{2}\right) s \int_{\nu} n(\nu) \mathrm{d} \nu,
$$

where $s$ is the geometrical cross-section of the laser beam.

One of the important questions one may raise, when reviewing the model presented above, concerns the absence of power broadening. In fact, power broadening could be expected to play an essential role, at least when tuning the pump rate, and consequently the laser power, to large values. In order to clarify this question it is helpful to call in mind that power broadening is not an elementary effect, but just the result of folding the actual population densities with the elementary lineshape of the transition. Large values of pump rate and power are capable of changing the population densities, but not the elementary lineshape of the transition. In other words, large values of pump rate and power have no direct impact on the lineshape itself, but a rather indirect one which is implicitly accounted for in our rate equations by tracing the dynamics of the population densities.

Now before moving on to applying the model on the example of He-Ne laser and analyzing the dynamics of lineshape and linewidth, it is worth highlighting what is actually new in the formalism presented above compared with the rate equations of [12]. Indeed, Eqs. (1),(2) are identical with the corresponding Eqs. (6),(7) of [12]. However, Eq. (3) distinguishes itself from the corresponding Eq. (8) of [12] by including a source term $\dot{n}_{\mathrm{sp}}(\nu) \mathrm{d} \nu$ which accounts explicitly for the change of the photon density due to spontaneous emission. This term, specified in Eq. (4), is of statistical nature and constitutes an incoherent source term which is completely missing in Eq. (8) of [12]. Furthermore, from a technical point of view, the resolution of the numerical calculation in [12] was limited to about $10 \mathrm{kHz}$ according to the reasonable limits on the computation time. This insufficiency has been removed in the present work by introducing a dynamical binning which allows for refining the resolution locally in the unsaturated regions of the spectrum while keeping the actual resolution unchanged in the saturated regions. This technique enabled a drastic improvement of the effective resolution from $10 \mathrm{kHz}$ down to values smaller than $1 \mathrm{~Hz}$.

\section{Results and discussion}

In our calculation we will refer to $\mathrm{He}-\mathrm{Ne}$ laser oscillating at $\lambda=632.8 \mathrm{~nm}$ since this type of laser had been subject of extensive studies [18-20] and is, therefore, most relevant for the purpose of comparison. The adopted values for the various parameters are listed in Table I. The gain is assumed to be homogeneously broadened with a reliable width of $50 \mathrm{MHz}$ involving the natural linewidth of $19 \mathrm{MHz}$ [25] as well as an additional contribution due to pressure broadening. The values for the parameters of the resonator are chosen to support single mode operation and to reproduce the cavity width of $4.7 \mathrm{MHz}$ reported in [20]. A closer analysis reveals, however, that single mode operation in a real $\mathrm{He}-\mathrm{Ne}$ laser requires the cavity length to be smaller than $11 \mathrm{~cm}$. So, strictly speaking, if we would consider not only the homogeneous but also the inhomogeneous broadening of the gain profile, our laser would oscillate not only at the central mode, but also at two additional, relatively weak, side modes separated by about $1 \mathrm{GHz}$ from the much stronger central mode. However, it can be argued that the consequences for the dynamics of the lineshape and linewidth - the main topic of the present study, as well as for the relationship between the linewidth and the power, are rather negligible, since the central mode is quite well separated from the side modes due to the large mode spacing of about 20 times the homogeneous width. On the other hand, it is worth noting once more that the validity of our rate equations is not restricted to a certain type of 
laser, and the model presented above can be considered applicable to different types of lasers with homogeneously broadened gain.

Adopted values for the model parameters.

TABLE I

\begin{tabular}{|c|c|c|c|}
\hline \multicolumn{2}{|l|}{ Parameter } & Value & Ref. \\
\hline $\begin{array}{l}\text { upper level - decay constant } \\
\text { degeneracy factor }\end{array}$ & $\begin{array}{c}A_{20} \\
g_{2}\end{array}$ & $\begin{array}{c}10^{6} \mathrm{~s}^{-1} \\
3\end{array}$ & $\begin{array}{l}{[21]} \\
{[22]}\end{array}$ \\
\hline $\begin{array}{c}\text { lower level - decay constant } \\
\text { degeneracy factor }\end{array}$ & $\begin{array}{c}A_{10} \\
g_{1}\end{array}$ & $\begin{array}{c}10^{7} \mathrm{~s}^{-1} \\
5\end{array}$ & {$\left[\begin{array}{l}{[21]} \\
{[22]}\end{array}\right.$} \\
\hline $\begin{array}{l}\text { Einstein coefficient for } \\
\text { the spontaneous transition } 2 \rightarrow 1\end{array}$ & $A_{21}$ & $3.4 \times 10^{6} \mathrm{~s}^{-1}$ & {$[23]$} \\
\hline $\begin{array}{c}\text { cross-section for the } \\
\text { stimulated transition } 1 \leftrightarrow 2\end{array}$ & $\sigma$ & $3 \times 10^{-13} \mathrm{~cm}^{2}$ & {$[24]$} \\
\hline cavity length & $l_{\mathrm{c}}$ & $14 \mathrm{~cm}$ & \\
\hline length of the active medium tube & $l_{\mathrm{m}}$ & $10 \mathrm{~cm}$ & \\
\hline mirror reflectivities & $\begin{array}{l}R_{1} \\
R_{2}\end{array}$ & $\begin{array}{c}1 \\
0.9724\end{array}$ & \\
\hline beam cross section & $s$ & $1 \times 10^{-3} \mathrm{~cm}^{2}$ & \\
\hline geometrical factor & $\xi$ & $5.3 \times 10^{-7}$ & \\
\hline
\end{tabular}

The initial conditions are chosen to match the experimental conditions prior to turning on the pumping, i.e. all atoms are assigned to the ground state, and accordingly, $M_{1}$ and $M_{2}$ are initialized to zero. As a result of the empty laser upper state, emission cannot occur and therefore, the photon density $n(\nu) \mathrm{d} \nu$ is also initialized to zero. Once the laser is turned on $(t=0)$, which is done by switching $\Phi$ to the actual value of the pump rate, $M_{2}, M_{1}$ and $n(\nu) \mathrm{d} \nu$ will start to change and are traced with time, step by step, until steady state values are established. In each time step, which is a small fraction of the round trip time, the actual values of $M_{1}, M_{2}$, and $n(\nu) \mathrm{d} \nu$ are used to calculate $G(\nu), \beta, \dot{n}_{\mathrm{sp}}(\nu) \mathrm{d} \nu, \dot{n}_{\mathrm{st}}(\nu) \mathrm{d} \nu$ and $\dot{n}_{\mathrm{c}}(\nu) \mathrm{d} \nu$ in sequence. These values are then used to calculate the actual changes of $M_{1}, M_{2}$, and $n(\nu) \mathrm{d} \nu$ according to the rate Eqs. (1)-(3). Finally, the new values of $M_{1}, M_{2}$ and $n(\nu) \mathrm{d} \nu$ are determined and made available for the next time step, and so on.

Calculations have been conducted for different values of the pumping rate $\Phi$. In all calculations, saturation of the overall power was reached within a faction of $\mu \mathrm{s}$. The saturated frequency spectrum was found to be featured by a single mode coinciding with the strongest cavity mode, a Lorentzian lineshape, and a linewidth much smaller than $\Gamma_{\mathrm{h}}$. For small values of the pumping rate $\Phi$, saturation of the frequency spectrum occurred more or less in time with the saturation of the overall power, and the saturated linewidth was close to the spectral width of the pure cavity $\Gamma_{\mathrm{c}}$. However, when $\Phi$ is increased above a certain threshold value $\Phi_{\mathrm{th}}$, the saturation time of the frequency spectrum turns out to dramatically exceed the saturation time of the power, and the linewidth is found to drop to extremely small values far below $\Gamma_{\mathrm{c}}$. This is illustrated in Fig. 1 by comparing the saturation curves of power and linewidth for two different values of the pumping rate below and above threshold. As seen in the figure, an increase of $\Phi$ by less than factor 10 causes the saturation time of the linewidth to increase by more than 7 orders of magnitude (from $0.5 \mu$ s to $20 \mathrm{~s}$ ) and the saturated linewidth to decrease by 4 orders of magnitude (from $4.2 \mathrm{MHz}$ to $400 \mathrm{~Hz}$ ), whereas the saturation time of the power changes only slightly by a factor of 1.5 (from $0.4 \mu \mathrm{s}$ to $0.6 \mu \mathrm{s})$.

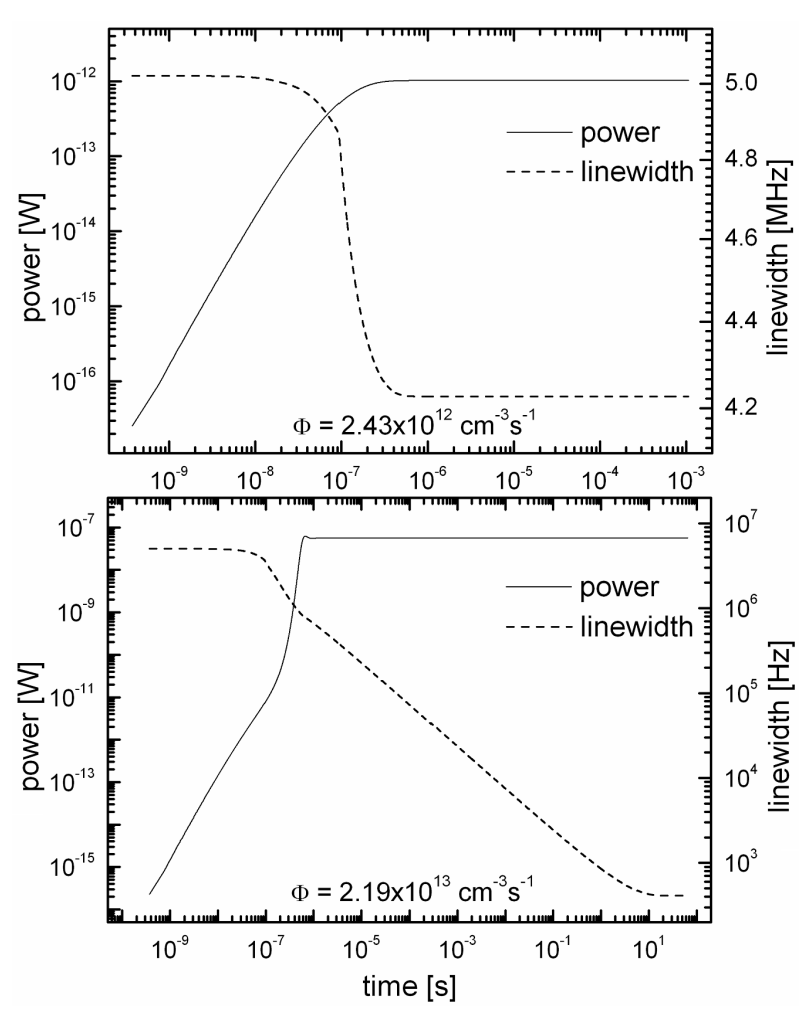

Fig. 1. Saturation dynamics of power and linewidth below (upper part) and above threshold (lower part).

First of all, it should be pointed out that the very long saturation time of the linewidth, when the laser is operated above threshold, may have substantial consequences for the laser frequency spectrum. It seems that a real laser would never have enough time to reach saturation of the frequency spectrum because there are always more restrictive time limits due to the technical noise, the limited pulse duration, as well as the limited temporal coherence. In other words, the process of line-narrowing will be disturbed at an earlier moment before the linewidth reaches its saturation value. Unfortunately, there are in the literature neither experimental nor theoretical data on the dynamics of laser linewidth to compare with. This is not surprising since, on the one hand, all available experimental methods for measuring the laser linewidth involve relatively long measurement times, and on the other hand, all existing theories for the laser linewidth consider the situation only under steady state conditions, i.e. after saturation has occurred.

Nevertheless, it is worth looking at the results presented in Fig. 1 from the point of view of our rate Eqs. (1)-(3). Indeed, the reason for the large difference between the saturation times of power and linewidth 
can be read from the rate equations themselves. From Eqs. (1) and (2), it is obvious that changes of the population densities are not coupled to the detailed structure of the spectrum, but only to the frequency-integrated rate of stimulated emission $\beta$. Therefore, saturation of the population densities, as well as of the overall power which is also an integral quantity, will not be affected by internal changes in the detailed structure of $n(\nu) \mathrm{d} \nu$ as long as the integrated photon density is unchanged. Indeed, power saturation is reached generally after a fraction of $\mu \mathrm{s}$, regardless of the value of $\Phi$. What follows after that involves exclusively the detailed structure of $n(\nu) \mathrm{d} \nu$, in particular the linewidth.

In order to get an insight into the physics behind the drastic change in the linewidth, as well as in its saturation time, when passing from below to above threshold, it is helpful to consider the laser frequency spectrum as superposition of two different components: a spontaneous spectrum settled by Eq. (4), and a stimulated spectrum satisfying Eq. (6). From Eq. (4), it is obvious that spontaneously emitted photons obey a stationary distribution proportional to $\mathcal{A}_{\mathrm{c}}(\nu) \mathcal{L}(\nu) \mathrm{d} \nu$ with a linewidth close to $\Gamma_{\mathrm{c}}$ (note that $\Gamma_{\mathrm{c}}$ is significantly smaller than $\Gamma_{\mathrm{h}}$ ). On the other hand, Eq. (6) tells us that stimulated photons do not follow a stationary, but a dynamic distribution proportional to the actual photon density $n(\nu) \mathrm{d} \nu$. Indeed, substituting $G(\nu)$ in Eq. (6) by its first order approximation according to Eq. (7) by making use of Eq. (8), one arrives at a non-stationary distribution proportional to $\mathcal{A}_{\mathrm{c}}(\nu) \mathcal{L}(\nu) n(\nu) \mathrm{d} \nu$ which is, in any case, narrower than $n(\nu) \mathrm{d} \nu$. And this means that the linewidth of the stimulated component will, necessarily, continue decreasing steadily with time approaching zero.

Now thinking about the laser frequency spectrum as composed of a spontaneous component with a linewidth close to $\Gamma_{\mathrm{c}}$ and a stimulated component with a dynamic linewidth which decreases with time towards zero, one can expect the laser linewidth to reflect the statistical average of the linewidths of both components. At the very early stage after starting the laser, photons are emitted exclusively by means of spontaneous decay, and the linewidth is determined by $\Gamma_{\mathrm{c}}$. As time goes on, inversion is built up, and the linewidth starts to decrease due to the increasing contribution of stimulated photons. Once power saturation is reached, the statistical weights of both spontaneous and stimulated components freeze at their actual values. From that point on, the spontaneous contribution to the linewidth is fixed, but the laser linewidth will still continue to decrease due to the decrease of linewidth of the stimulated component. Saturation of the linewidth will be established when the weighted stimulated width will have reached values significantly smaller than the weighted spontaneous width. Thus, it can be stated that the saturation value of the linewidth, as well as its saturation time, are mainly determined by the statistical weight of the spontaneous component. Below threshold, the statistical weight of spontaneous photons is close to unity, and consequently, the linewidth is close to $\Gamma_{\mathrm{c}}$ and the saturation time of the linewidth is close to that of the power (upper part of Fig. 1). Above threshold however, the statistical weight of spontaneous photons is very small compared to unity, and therefore, the linewidth is a very small fraction of $\Gamma_{\mathrm{c}}$ and the saturation time of the linewidth is much larger than that of the power (lower part of Fig. 1).

At this point, it is worth pointing out why we specify the threshold point in terms of the threshold pump rate rather than the threshold inversion commonly used in the literature. Actually, most of the classical works on laser operation describe the threshold condition by setting the total round-trip gain - i.e. the amplification due to stimulated emission multiplied by the attenuation due to the cavity losses - equal to unity [25, 26] arriving at a relation between threshold inversion and loss factor of the cavity. However, we believe that this characterization is questionable, because it considers the balance between stimulated emission and cavity losses neglecting the contribution of spontaneous emission, which is expected to be comparable to stimulated emission in the threshold region. Moreover, in contrast to the inversion, the pump rate is a directly accessible control parameter which can be easily adjusted to control the gain. For these reasons, we specify the threshold point by the value of pump rate at which stimulated emission starts to dominate over spontaneous emission.

We have computed the rate of spontaneous, as well as stimulated emission, over a wide range of the pumping rate $\Phi$. The results are plotted in the lower part of Fig. 2, the corresponding values of the overall power are plotted in the upper part of the figure. It is obvious from Fig. 2 that spontaneous emission clearly dominates over stimulated emission in the low pump rate region. However, the rate of stimulated emission increases more rapidly with increasing pump rate, and reaches the rate of spontaneous emission at a certain pump rate $\Phi=8.5 \times 10^{12} \mathrm{~cm}^{-3} \mathrm{~s}^{-1}$ which we refer to as the threshold pump rate $\Phi_{\mathrm{th}}$. Immediately after passing the threshold pump rate $\Phi_{\mathrm{th}}$, stimulated emission undergoes a very steep increase at the expense of a similarly steep decrease of spontaneous emission. Note that the rate of stimulated emission increases by more than four orders of magnitude within the very narrow region between $\Phi_{\mathrm{th}}$ and twice $\Phi_{\mathrm{th}}$ which will be referred to in the following as the threshold region. After leaving the threshold region, stimulated emission continues to increase steadily with increasing $\Phi$, whereas spontaneous emission passes first through an intermediate region, in which it recovers slowly from the decrease, and then resumes increasing with increase of $\Phi$.

The upper part of Fig. 2 illustrates that the laser power simply follows the dominant component of the two emission rates. Below threshold, the power is proportional to the rate of spontaneous emission, whereas above threshold, it is proportional to the rate of stimulated emission, and accordingly, it experiences an increase by more than four orders of magnitude within the borders of the narrow threshold region. 

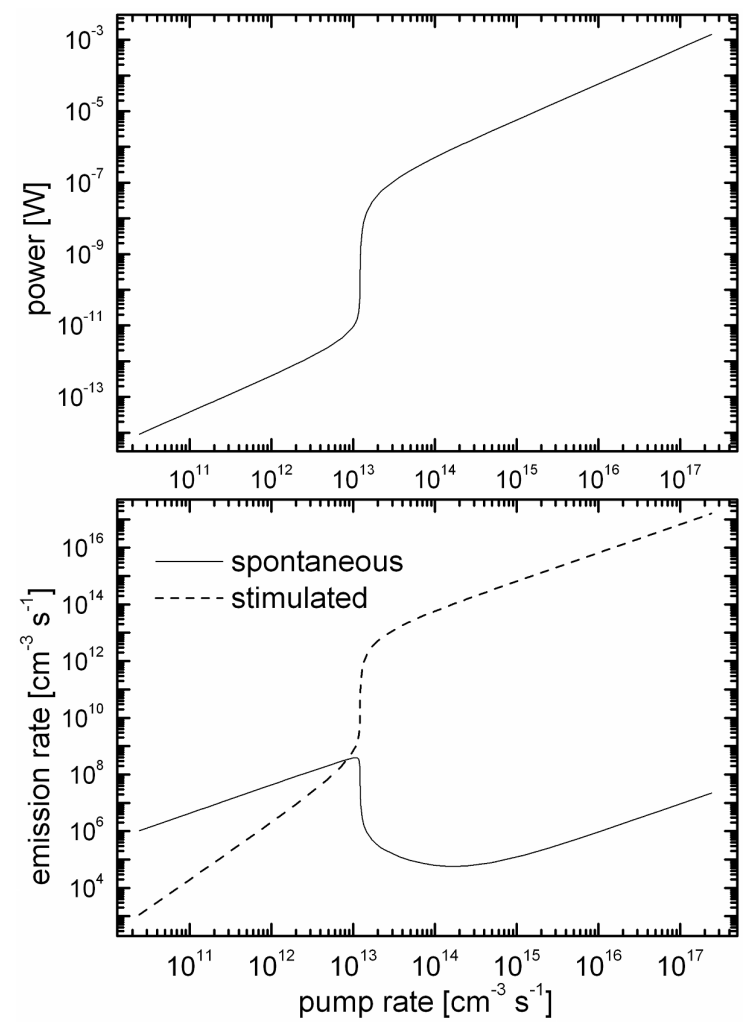

Fig. 2. Threshold characteristics of the power (upper part) as well as the emission rates for spontaneous and stimulated photons (lower part).

The next issue to be considered in this dynamical study of the laser frequency spectrum is the dynamics of the lineshape. We have revealed in advance, quite at the beginning of this section, that the saturated lineshape is found to be Lorentzian. However, thinking about the relatively long saturation time for the laser frequency spectrum, which is expected to exceed the coherence time for many types of laser systems, it is absolutely worth examining not only the saturated but also the unsaturated lineshape. We have observed the time evolution of the lineshape from the moment of starting the laser to the very late stage of ultimate saturation. The results can be summarized as follows. The line profile starts with a Lorentzian shape and a width close to the cavity width $\Gamma_{\mathrm{c}}$ and ends up also with a Lorentzian shape, but with a linewidth much smaller than $\Gamma_{\mathrm{c}}$. However, the intermediate line profile deviates significantly from the Lorentzian shape. It looks closer to a Gaussian in the peak region, but shares, at the same time, the far tail of the saturated line profile.

Figure 3 gives an illustration of the saturation behaviour of the line profile. The figure shows two different snapshots of the unsaturated line profile, taken at 0.1 and $0.5 \mathrm{~s}$, in comparison with the Lorentzian-shaped saturated line profile established after about $20 \mathrm{~s}$. On the basis of Fig. 3, the overall saturation behaviour of the line profile can be sketched as follows. Saturation occurs
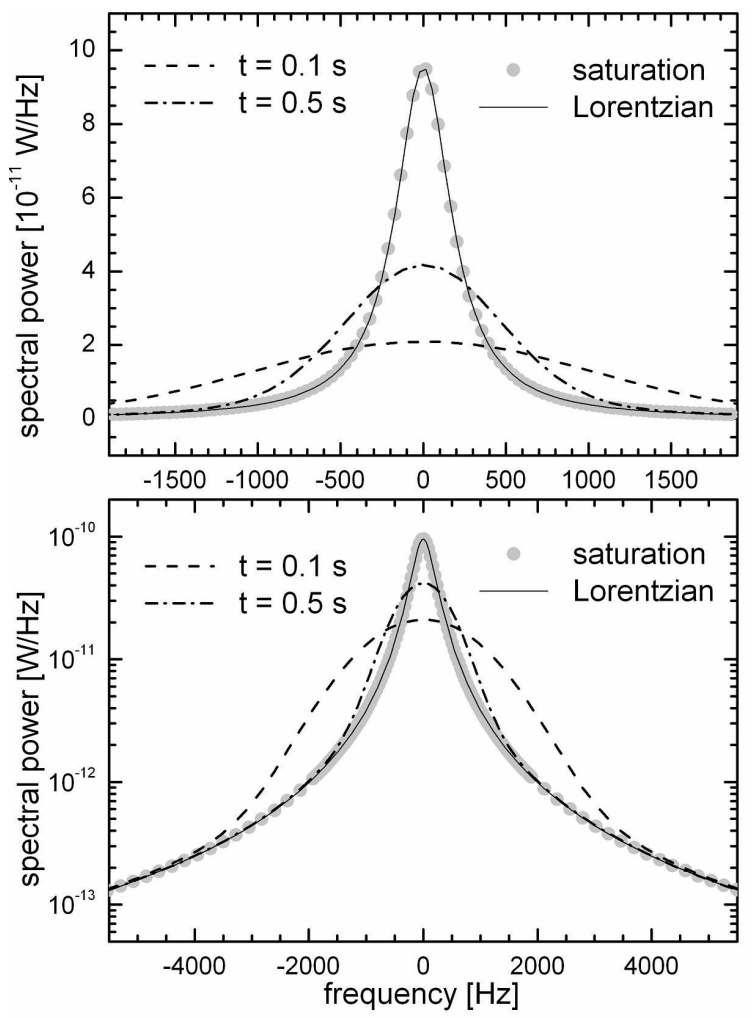

Fig. 3. Saturation dynamics of the lineshape. Snapshots of the line profile are shown in a linear (upper part) and a logarithmic plot (lower part) in order to depict both the peak and its far tail.

first in the far tail, and moves slowly towards the centre of the line. In fact, this process shares the same origin as the saturation process of the linewidth discussed above in connection with Fig. 1.

Finally, let us move on to the question of the relationship between laser linewidth and laser power, a question which has been subject of numerous theoretical as well as experimental investigations. Having the advantage of being capable to calculate the linewidth below, around, and above threshold, our model is best suited for a comprehensive and independent investigation of the linewidth vs. power relationship.

In a long series of runs, the saturated linewidth has been computed over the complete significant range of laser power, starting at a very low power, 3 orders of magnitude below the threshold power $P_{\mathrm{th}}=8.4 \times 10^{-12} \mathrm{~W}$, and stopping at an extremely high power, 8 orders of magnitude above $P_{\mathrm{th}}$. The results are plotted in Fig. 4 in comparison with the theoretical values (ST and STsimplified) predicted according to the Schawlow-Townes formula

$$
\Delta \nu=\frac{\pi h \nu_{0} \Gamma_{\mathrm{c}}^{2}}{P} \frac{M_{2}}{M_{2}-\left(g_{2} / g_{1}\right) M_{1}},
$$

in its entire form, respectively in its widely used simplified form in which the population factor - last factor in Eq. (12) - is fixed to unity [20]. Experimental results from [19] and [20] are also included in the figure. 

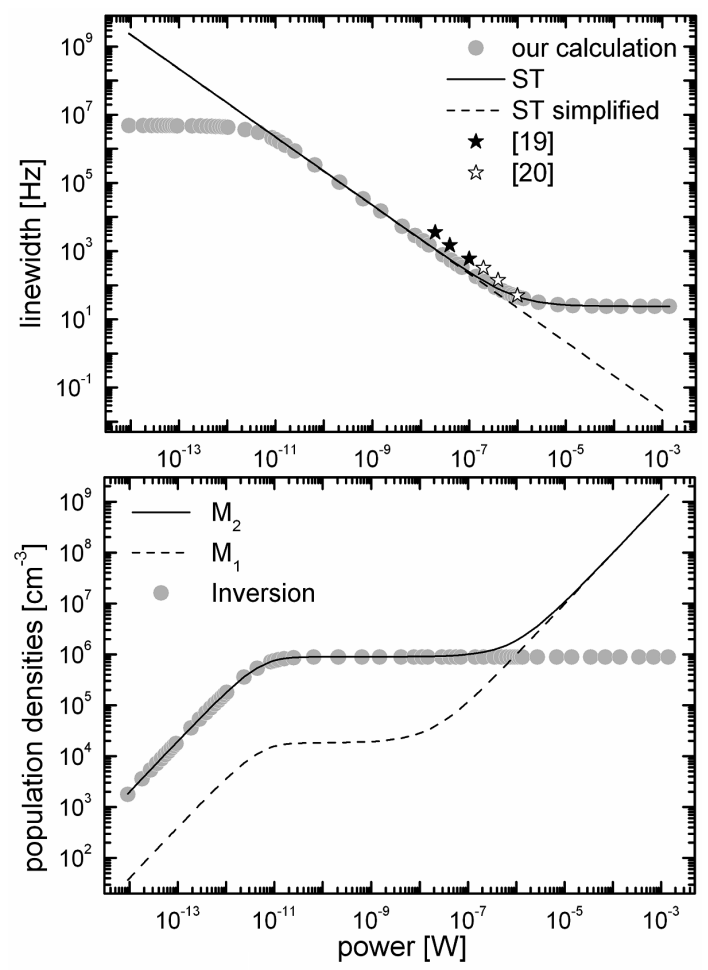

Fig. 4. Linewidth vs. laser power in comparison with the theoretical predictions according to the ST-formula, as well as the experimental results of [19] and [20] (upper part). The corresponding values for the population densities are plotted in the lower part in order to illustrate the validity limit of the simplified ST-formula.

Considering the above-threshold region in Fig. 4, it can be argued that our results agree quite well with Eq. (12) and, to within a factor 2 , with the experimental results of [19] and [20]. However, a closer analysis shows that the linewidth computed by our model should not be directly compared with Eq. (12), but only to within a scale factor determined by the values of the parameters $s$ and $\xi$ which are not involved in Eq. (12). The values of $s$ and $\xi$ given in Table I were chosen to achieve best agreement with Eq. (12). In the below-threshold region, our linewidth approaches an upper limit defined by the cavity width $\Gamma_{\mathrm{c}}$, whereas the linewidth generated by Eq. (12) continues increasing unlimitedly with decrease of power. In fact, we believe that the behaviour of the STlinewidth in the below-threshold region, particularly its unlimited increase towards values exceeding not only the cavity width but also the source width, is not reasonable. Moreover, in the original works of Schawlow and Townes, Eq. (12) was derived by considering the laser as an amplifier with a weak input due to thermal radiation [27], or to spontaneous emission [5], and a much stronger output due to stimulated emission. This basic condition is by far not fulfilled below threshold where spontaneous emission constitutes the dominant component of the output radiation (see Fig. 2, lower part).

Another exciting behaviour of our calculated linewidth manifests itself in a deviation from the $1 / P$ dependence, with a strong tendency to approach a lower limit, when increasing the laser power far above the threshold. Note that this behaviour is not an exclusive result of our numerical calculations based on the rate Eqs. (1)-(3). It is also perfectly reproduced by Eq. (12) when adopting our saturation values for the population densities (solid line in the upper part of Fig. 4), whereas the simplified STformula (dashed line in the upper part of Fig. 4) supports the common picture of an unlimited decrease of the linewidth with increase of power. This means that the observed deviation from the $1 / P$ dependence can be directly ascribed to a considerable deviation of the population factor from unity. According to Eq. (12), the $1 / P$ behaviour of the linewidth corresponds to a constant value of the population factor, whereas a lower limit of the linewidth indicates a linear increase of the population factor with increasing power. In order to understand the behaviour of population factor when moving towards the far-above-threshold region, the population densities $M_{1}$ and $M_{2}$, as well as the inversion, are plotted as functions of the laser power in the lower panel of Fig. 4. Now let us trace the changes of $M_{1}$ and $M_{2}$ trying to reconstruct the behaviour of the population factor, as well as of the linewidth, in the different regions along the power axis of Fig. 4. It is quite helpful to distinguish between the following four regions:

(i) below threshold $\left(P<10^{-11} \mathrm{~W}\right): M_{1}$ and $M_{2}$ increase steadily with increasing power, $M_{2}$ is larger than $M_{1}$ since the life time of the laser upper state is longer than the life time of the laser lower state, inversion builds up but is still too small to make stimulated emission dominant over spontaneous emission, and thus the spectrum is dominated by the spontaneous component (see Fig. 2), the linewidth has a more or less constant value close to the cavity width, and the ST-formula is not applicable;

(ii) threshold region $\left(10^{-11}-10^{-8} \mathrm{~W}\right)$ : inversion has reached its saturation value, the spectrum is now dominated by the stimulated component, increasing the power over this region requires only a slight increase of the pump rate (see Fig. 2) associated with a rather negligible increase of $M_{1}$ and $M_{2}$, the population factor keeps a constant value close to unity, and the linewidth follows the famous $1 / P$ dependence;

(iii) near-threshold region $\left(10^{-8}-10^{-6} \mathrm{~W}\right)$ : increasing the power over this region requires a considerable increase of the pump rate (Fig. 2) associated with a reasonable increase of the population densities, both $M_{1}$ and $M_{2}$ increase by the same value in order to keep the inversion unchanged, however, since $M_{1}$ is much smaller than $M_{2}$, the relative increase of $M_{1}$ is large, whereas $M_{2}$ can still be considered nearly constant as readily seen in Fig. 4, and consequently, the population factor in Eq. (12) can still be well approximated to unity, and the linewidth still follows the $1 / P$ dependence; 
(iv) far-above-threshold region $\left(P>10^{-6} \mathrm{~W}\right): M_{1}$ has reached a value comparable to $M_{2}$, further increase of the power, by means of further increasing the pump rate, is associated with a linear increase of both $M_{1}$ and $M_{2}$ whereas the inversion is still keeping its saturation value, the population factor in Eq. (12) is, therefore, no more constant but increases linearly with increasing power, and consequently, the linewidth approaches a constant value.

For the sake of completeness it should be noted here that the pump rate values needed to explore the farabove-threshold region are excessively large, and hardly accessible in the experiment. Hence, the observed deviation from the simplified ST-formula may be considered, for the time being, of purely theoretical importance.

\section{Conclusions}

We have studied the dynamics of laser lineshape and linewidth by means of numerical calculations based on solving the rate equations in both time and frequency domains. It has been found that the saturation of the frequency spectrum is by several orders of magnitude slower than the saturation of the overall power. The saturated lineshape proved to be Lorentzian, whereas the unsaturated line profile turned out to combine a Gaussian peak together with the saturated Lorentzian tail. To our knowledge, the question of the dynamics of laser lineshape and linewidth has never been addressed before. Thus, our model is the first of its kind to provide a powerful tool for studying the dynamics of laser lineshape and linewidth. One of the important applications would be to calculate the frequency spectrum for pulsed lasers.

Furthermore, we have applied our numerical model to investigate the relationship between laser linewidth and laser power. Our linewidth proved to be in good agreement with the Schawlow-Townes formula over the whole above-threshold region. Below the threshold, however, our linewidth approaches an upper limit defined by the spectral width of the pure cavity, in contradiction to the widely accepted prediction of semiclassical theories according to which the ST-formula is said to remain applicable below the threshold after multiplying the right hand side by the scale factor 2. Moreover, our calculations revealed that the inverse proportion between laser linewidth and laser power holds only up to a certain power. Far above threshold, our linewidth is found to approach a lower limit due to a linear increase of the population factor with increasing power, and this finding is supported by the ST-formula itself when adopting the actual population densities, and is contradictory to the simplified ST-formula according to which the linewidth would continue decreasing unlimitedly with increase of power.

\section{Acknowledgments}

This study was supported by the Atomic Energy Commission of Syria.

\section{References}

[1] G.S. Hurst, M.G. Payne, Principles and Application of Resonance Ionisation Spectroscopy, Adam Hilger, Bristol 1988.

[2] G.P. Gupta, B.M. Suri, Phys. Rev. A 77, 23419 (2008).

[3] T. Arisawa, Y. Maruyama, Y. Suzuki, Y. Naruse, J. Phys. D 16, 2415 (1983).

[4] M. Sankari, P.V.K. Kumar, M.V. Suryanarayana, J. Phys. B 33, 4927 (2000).

[5] A.L. Schawlow, C.H. Townes, Phys. Rev. 112, 1940 (1958).

[6] W.E. Lamb Jr., Phys. Rev. A 134, 1429 (1964).

[7] M. Scully, W.E. Lamb Jr., Phys. Rev. Lett. 16, 853 (1966).

[8] M. Lax, Phys. Rev. 129, 2342 (1963).

[9] R.D. Hempstead, M. Lax, Phys. Rev. 161, 350 (1967).

[10] H. Haken, Laser Theory, Springer, Berlin 1984.

[11] H.J. Carmichael, in: Lasers and Quantum Optics, Eds. L.M. Narducci, E.J. Quel, J.R. Tredicce, World Sci., Singapore 1988, p. 52.

[12] M. Eskef, Acta Phys. Pol. A 121, 599 (2012).

[13] I. Burak, P.L. Houston, D.J. Sutton, J.I. Steinfeld, IEEE J. Quant. Electron. QE-7, 73 (1971).

[14] W.W. Rigrod, J. Appl. Phys. 36, 2487 (1965).

[15] Y.J. Kaufman, U.P. Oppenheim, Appl. Opt. 13, 374 (1974).

[16] G. Gabrielse, H. Dehmelt, Phys. Rev. Lett. 55, 67 (1985).

[17] E.M. Purcell, H.C. Torrey, R.V. Pound, Phys. Rev. 69, 37 (1946).

[18] A.E. Siegman, R. Arrathoon, Phys. Rev. Lett. 20 , 901 (1968).

[19] R. Arrathoon, A.E. Siegman, J. Appl. Phys. 40, 910 (1969).

[20] H. Gerhardt, H. Welling, A. Güttner, Z. Phys. 253 , 113 (1972).

[21] C.K. Rhodes, A. Szöke, in: Laser Handbook, Eds. F.T. Arecchi, E.O. Schultz-DuBois, NorthHolland Publ., Amsterdam 1972, p. 265.

[22] K. Burns, K.B. Adams, J. Longwell, J. Opt. Soc. Am. 40, 339 (1950).

[23] J.R. Fuhr, W.L. Wiese, in: CRC Handbook of Chemistry and Physics, Ed. D.R. Lide, CRC Press, Florida 1998, p. 10.

[24] W.T. Silfvast, Laser Fundamentals, Cambridge University Press, Cambridge 2004.

[25] O. Svelto, Principles of Lasers, Springer, New York 2010, p. 434.

[26] A.E. Siegman, Lasers, Mill Valley, California 1986 , p. 39.

[27] J.P. Gordon, H.J. Zeiger, C.H. Townes, Phys. Rev. 99, 1264 (1955). 